\title{
Alexa Conversations: An Extensible Data-driven Approach for Building Task-oriented Dialogue Systems
}

\author{
Anish Acharya, Suranjit Adhikari, Sanchit Agarwal, Vincent Auvray, Nehal Belgamwar, \\ Arijit Biswas, Shubhra Chandra, Tagyoung Chung, Maryam Fazel-Zarandi, Raefer Gabriel, \\ Shuyang Gao, Rahul Goel, Dilek Hakkani-Tur, Jan Jezabek, Abhay Jha, Jiun-Yu Kao, \\ Prakash Krishnan, Peter Ku, Anuj Goyal, Chien-Wei Lin, Qing Liu, Arindam Mandal, \\ Angeliki Metallinou, Vishal Naik, Yi Pan, Shachi Paul, Vittorio Perera, Abhishek Sethi; \\ Minmin Shen, Nikko Strom and Eddie Wang \\ Amazon Alexa AI, Sunnyvale, California, $\mathrm{USA}^{\dagger}$
}

\begin{abstract}
Traditional goal-oriented dialogue systems rely on various components such as natural language understanding, dialogue state tracking, policy learning and response generation. Training each component requires annotations which are hard to obtain for every new domain, limiting scalability of such systems. Similarly, rule-based dialogue systems require extensive writing and maintenance of rules and do not scale either. End-to-End dialogue systems, on the other hand, do not require module-specific annotations but need a large amount of data for training. To overcome these problems, in this demo, we present Alexa Conversations ${ }^{1}$, a new approach for building goal-oriented dialogue systems that is scalable, extensible as well as data efficient. The components of this system are trained in a data-driven manner, but instead of collecting annotated conversations for training, we generate them using a novel dialogue simulator based on a few seed dialogues and specifications of APIs and entities provided by the developer. Our approach provides outof-the-box support for natural conversational phenomena like entity sharing across turns or users changing their mind during conversation without requiring developers to provide any such dialogue flows. We exemplify our approach using a simple pizza ordering task and showcase its value in reducing the developer burden for creating a robust experience. Finally, we evaluate our system using a typical movie ticket booking task and show that the dialogue simulator is an essential component of the system that leads to over $50 \%$ improvement in turn-level action signature prediction accuracy.
\end{abstract}

\section{Introduction}

Goal-oriented dialogue systems enable users to complete specific goals such as making restau-

\footnotetext{
${ }^{*}$ Work done while at Amazon

${ }^{\dagger}$ Authors are ordered alphabetically

${ }^{1}$ https://tinyurl.com/y3lowd34
}

rant reservations and buying train tickets. User goals may be complex and may require multiple turns to achieve. Moreover, users can refer to contextual values anaphorically, can correct previously informed preferences and provide additional or fewer entities (over-cooperative or undercooperative user) than requested by the agent. This presents challenges for building robust dialogue agents that need to understand different kinds of user behavior, gather user requirements split over multiple turns and complete user goals with minimal friction. There is also limited availability of dialogue datasets and they span only a handful of application domains. Designing suitable data collection for dialogue systems is itself a research area.

Traditional dialogue systems follow a pipelined approach that ties together machine learning components for natural language understanding (NLU), dialogue state (belief) tracking, optimal action prediction (policy learning), and natural language generation (Young, 2000). Advances in deep learning techniques have led to the development of more end-to-end neural dialogue systems that combine some or all of the components of the traditional pipeline reducing the need for component-wise annotations and allowing for intermediate representations to be learned and optimized end-to-end (Wen et al., 2017; Liu et al., 2017). On the data side, notable data collection approaches for dialogue systems include the Wizard-of-Oz (WOZ) framework (Asri et al., 2017), rule-based or data-driven user simulators (Pietquin, 2005; Cuayáhuitl et al., 2005; Pietquin and Dutoit, 2006; Schatzmann et al., 2007; Fazel-Zarandi et al., 2017; Gur et al., 2018), and the recently-proposed Machines-Talking-To-Machines (M2M) framework (Shah et al., 2018) where user and system simulators interact with each other to generate dialogue outlines.

In this demo, we present Alexa Conversations, a novel system that enables developers to build ro- 
bust goal-oriented dialogue experiences with minimal effort. Our approach is example-driven as it learns from a small number of developer-provided seed dialogues and does not require encoding dialogue flows as rigid rules. Our system contains two core components: a dialogue simulator that generalizes input examples provided by the developer and a neural dialogue system that directly predicts the next optimal action given the conversation history. The dialogue simulator component extends the M2M framework (Shah et al., 2018) in two main directions. First, instead of generating user goals randomly, we use various goal sampling techniques biased towards the goals observed in the seed dialogues in order to support variations of those dialogues robustly. Second, in $\mathrm{M} 2 \mathrm{M}$, the system agent is geared towards database querying applications where the user browses a catalogue, selects an item and completes a transaction. In contrast, our formulation does not require any knowledge of the purpose of the APIs provided by the developer. Moreover, our system can generate a richer set of dialogue patterns including complex goals, proactive recommendations and users correcting earlier provided entities. The proposed neural dialogue model component follows an endto-end systems approach and bears some similarities with Hybrid Code Networks (HCN) (Williams et al., 2017). However, compared to HCN, our system is more generic in the sense that it directly predicts the full API signature that contains the API name, values of the required API arguments, relevant optional API arguments and their values. The model chooses the API argument values to fill from user mentioned, agent mentioned and API returned entities present in the full dialogue context that includes the current user utterance.

We showcase the significance of our approach in reducing developer burden using the example of a pizza ordering skill. Compared to a rule-based system where a developer would have to code hundreds of dialogue paths to build a robust experience even for such a simple skill, Alexa Conversations requires only a handful of seed dialogues. To evaluate our approach, we build a movie ticket booking experience. On a test set collected via Wizardof-Oz (WOZ) framework (Asri et al., 2017), we quantify the impact of our novel dialogue simulation approach showing that it leads to over $50 \%$ improvement in action signature prediction accuracy.

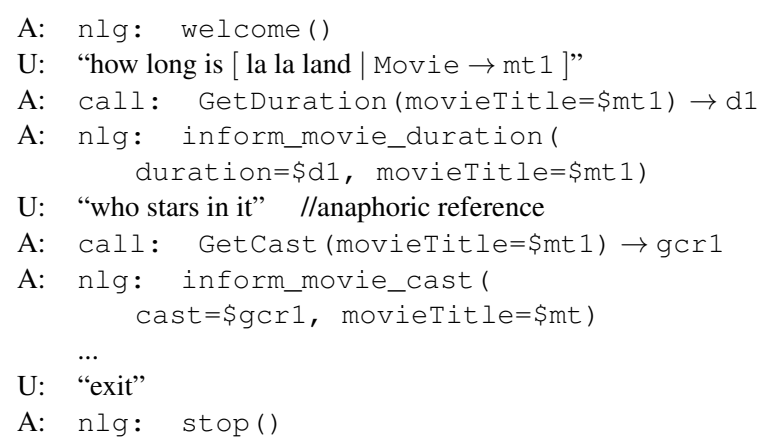

Table 1: A seed dialogue with DML annotations. Note that variables are carried over to resolve anaphoric references.

\begin{tabular}{|c|c|}
\hline Template Name & Template Text \\
\hline $\begin{array}{l}\text { inform_movie_duration } \\
\text { inform_movie_cast } \\
\text { offer_recommend_movie }\end{array}$ & $\begin{array}{l}\text { "\$movieTitle is \$duration long" } \\
\text { "\$cast.name was in \$movieTitle" } \\
\text { "Would you like a \$genre movie?" }\end{array}$ \\
\hline
\end{tabular}

Table 2: Developer-provided system NLG responses

\section{System Overview}

In Alexa Conversations, we follow a data-driven approach where the developer provides seed dialogues covering the main use cases they want to support, and annotates them in a Dialogue Markup Language (DML). Table 1 shows an example of an annotated conversation. Developers are required to provide their domain-specific APIs and custom Natural Language Generation (NLG) responses for interacting with the user, e.g., for informing an API output response or for requesting an API input argument as shown in Table 2. These APIs and system NLG responses, with their input arguments and output values, define the domain-specific schema of entities and actions that the dialogue system will predict. Developers also provide example userutterances (as templates with entity-value placeholders) which the users may use to invoke certain APIs or to inform slot values.

To handle the wide variation of conversations a user can have with the dialogue system, Alexa Conversations augments the developer provided seed dialogues through a simulator. This component takes the annotated seed dialogues as input, and simulates different dialogue flows that achieve the same user goals but also include common patterns such as when a user confirms, changes, or repeats an entity or action. Optionally, it uses crowdsourcing through Amazon Mechanical Turk (MTurk) to enrich the natural language variations of user utterances provided by the developer. Overall, the 


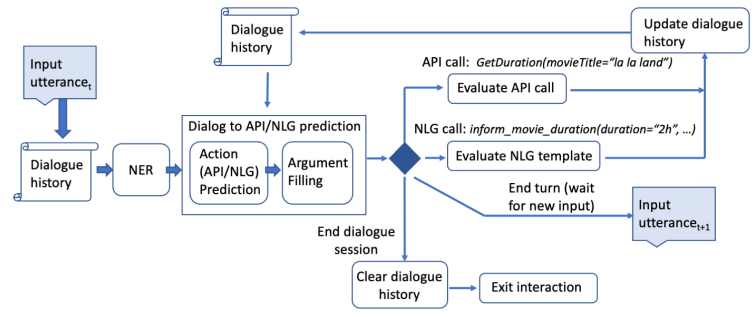

Figure 1: High-level overview of an input utterance's path

developer provides on the order of 10 seed dialogues and the simulator generates on the order of $10 \mathrm{~K}$ training dialogues with flow and language variations.

Alexa Conversations consists of three main domain-specific modeling components: 1) a Named-Entity Recognition (NER) model that tags entities in the user utterance (e.g., "La La Land" as a MovieTitle), 2) an Action Prediction (AP) model that predicts which API or NLG response should be executed next (e.g., GetDuration or inform_movie_duration), and 3) an Argument Filling (AF) model that fills required (and possibly optional) action arguments with entities (e.g., GetDuration(MovieTitle= "La La Land")). We use the entire dialogue history, i.e., user utterances, system actions and responses, and API return values, as input for all modeling components. In this sense, this dialogue history is used as a generalized state representation from which models can retrieve relevant information. An overview of the runtime flow of a dialogue is illustrated in Figure 1. Each user utterance initiates a turn and is followed by NER, after which one or more actions are predicted. These actions could be either an API or NLG call, or a special action indicating the end of a turn or the end of dialogue. Every new action prediction updates the dialogue history and therefore influences future action predictions. For each API/NLG call the AF model is called to fill in the required arguments. When <end of turn $>$ is predicted, the system waits for new user input. When $<$ end of dialogue $>$ is predicted, the system ends the interaction.

\section{Dialogue Simulation}

We propose a novel component called simulator to generate diverse but consistent dialogues, which can be used to train robust goal-oriented neural dialogue systems. We presented the simulator details

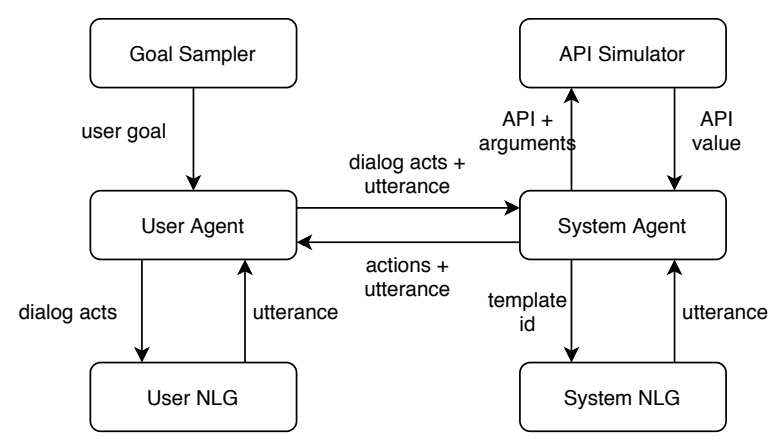

Figure 2: Simulator Architecture

in (Lin et al., 2020) and briefly provide an overview of the overall system here. A high-level simulator architecture is illustrated in Figure 2.

The simulator is structured in two distinct agents that interact turn-by-turn: the user and the system. The user samples a fixed goal at the beginning of the conversation. We propose novel goal-sampling techniques (Lin et al., 2020) to simulate variation in dialogue flows. The agents communicate at the semantic level through dialogue acts. Having the exact information associated with each turn allows us to define a simple heuristic system policy, whose output can be used as supervised training labels to bootstrap models. We note that the user policy is also heuristic-based. In each conversation, the user agent gradually reveals its goal and the system agent fulfills it by calling APIs. The system agent simulates each API call by randomly sampling a return value without actually calling the API and chooses an appropriate response action. Depending on the returned API value, the chosen response is associated with dialogue acts. The system agent gradually constructs an estimate of the user goal and makes proactive offers based on this estimated goal. The dialogue acts generated through self-play are also used to interface between agents and their template-based NLG model. After sampling the dialogue acts from their policy, each agent samples the surface-form from available templates corresponding to the dialogue acts. In addition to enriching the dialogue flows; we use crowd-sourcing through MTurk to enrich the natural language variations of the user utterance templates. Goal sampling and the self-play loop provide dialogue flow variations while crowd-sourcing enriches language variations, both of which are essential for training robust conversational models.

We introduce additional variations to dialogues during simulation for more natural conversation 


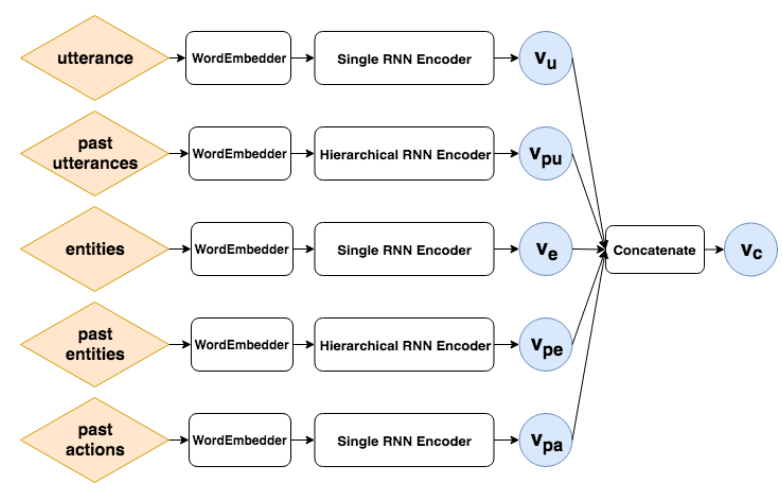

Figure 3: An example of a dialogue context encoder. Different downstream models use slightly different subsets of these features as input.

generation. In goal-oriented conversations, users often change their mind during the course of the conversation. For example, while booking a movie ticket a user may decide to purchase three adult tickets but could eventually change their mind to book only two tickets. We used additional heuristics to introduce such variations to conversations without any additional input requirements from the developer. Another important non-task-specific conversational behavior is the system's ability to suggest an appropriate next action based on the conversation history, without requiring invocation by a specific user utterance. We introduce proactive offers in the system policy of the simulator to facilitate exploration of the available API functionality in a manner consistent with human conversation.

\section{Models}

For each domain, we have three separate models: NER, Action Prediction (AP) and Argument Filling (AF), all of which depend on features extracted from conversation history and encoded using Dialogue Context Encoders.

\subsection{Dialogue Context Encoders}

Given a dialogue, we first apply feature extractors to extract both turn-level, e.g. current_user_utterance and current_entities (recognized by the NER model), and dialogue-level features, e.g. past_user_utterances, past_actions and past_entities. We pass these extracted features through feature-specific encoders and concatenate the feature representations to obtain the final representation for dialogue context. For encoding turn-level features and dialogue-level features, we use single LSTM and hierarchical
LSTM architectures, respectively. For example, for encoding past_user_utterances, we use a hierarchical LSTM, where we encode the sequence of words with an inner LSTM and the sequence of turns with an outer LSTM. For past_actions, a single LSTM is sufficient. Figure 3 shows an example of our dialogue context encoders. We augment the context encoders with word and sentence embedding vectors from pre-trained language models (Peters et al., 2018; Devlin et al., 2018).

\subsection{NER}

The NER model is used to extract domain-specific entities from user utterances, which are then consumed by downstream models. Our NER model is based on bi-LSTM-CRF (Ma and Hovy, 2016) model. To incorporate dialogue history, we concatenate the encoded dialogue context to the word embedding of each token and use it as the input to our model. To improve NER performance on entities with large and dynamic possible values (e.g. movie titles, restaurant names), we also incorporate catalogue-based features based on domain-specific catalogues of entity values provided by the developer and values returned by APIs. Specifically, catalogue features are computed by scanning the utterance with consecutive windows of size $n$ tokens and detecting any exact matches of the current window with the catalogue entries. For a domain with $K$ domain-specific catalogues, the binary feature will be of dimension $K$, where value 1 indicates an exact match in the catalogue. This approach is inspired by (Williams, 2019), which proposed a generic NER approach but not specific to conversational systems.

\subsection{Action Prediction (AP)}

The goal of the Action Prediction model is to predict the next action the agent should take, given the dialogue history. As illustrated in Figure 1, an action could be an API name (e.g. GetDuration), a system NLG response name (e.g. inform_movie_duration) or a general system action (e.g. <end of $\operatorname{turn}>$ ). The model takes the dialogue context encoding, as described in Section 4.1 and passes it through linear and softmax layers to output a distribution over all actions within the domain. Our system selects $n$ best action hypotheses using a simple binning strategy. We reject actions in the low confidence bins and if there is no actions available in the high- 


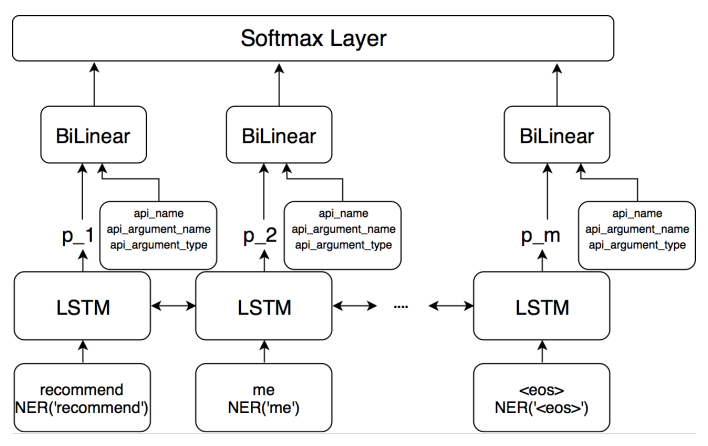

Figure 4: Argument filling model architecture

confidence bin, we randomly sample an action from the medium-confidence bin.

\subsection{Argument Filling (AF)}

The role of the Argument Filling model is to fill the arguments given a particular action and the dialogue history. We formulate the argument filling task as a variation of neural reading comprehension (Chen, 2018) where we treat the dialogue history as a passage to comprehend and ask machine the question "what is the argument value of a particular action?". Specifically, for each argument of an action and each entity mention detected by NER, our model predicts whether to use that entity mention to fill that argument. We do this by encoding all the entities in the dialogue history and use a pointer mechanism to point to the entity position given a particular action and argument combination. The overall architecture for argument filling is shown in Figure 4. Note that a similar method to dialogue state tracking has been proposed by (Gao et al., 2019). We impose constraints to only fill arguments with entities of the correct type according to the action schema provided by the developer. For example, we only consider Time entity mentions to fill arguments that accept Time type. Finally, we combine the argument filling result with the action prediction result to execute the next system action. API arguments can also be optional. For these type of arguments, we create special optional token and insert in the dialogue context and train the model to point to that token when an optional API argument should not be filled.

\section{Case Study 1: Pizzabot}

To showcase how Alexa Conversations improves the skill-building experience of a typical developer, we discuss Pizzabot, a pizza ordering skill. Pizzabot is provided as one of the template Alexa Con- versations skills to help bootstrap a new developer. It allows a user to order a pizza of various sizes, with different kinds of crusts and their choice of any number of toppings. The skill has just one main API OrderPizza with four slots types: toppingsList, crust, cheese and size. Even with this simple skill, ordering a two topping pizza with a choice of say 10 toppings, 5 crusts and 5 cheese in 4 different sizes results in over hundreds of different possible dialogue paths. With a typical rule-based dialogue manager, a developer would need to code most, if not all, of these flows for building a robust experience. On the other hand, with Alexa Conversations, a Pizzabot developer needs to provide just 7 example dialogues to support a total of 3 APIs and 6 entity types. The different variations are automatically generated by the proposed simulator and are consumed by the models, resulting in a robust skill with significantly lower developer effort. Alexa Conversations also provides out-of-the-box support for features like context carryover, entity confirmation, user correction, over/under-cooperative user, and multi-value slots without requiring the developer to write custom rules or logic, making the skill more robust to real user interactions. See Table 3 for an example interaction with Pizzabot.

U: I want to order a large pizza

A: You wanted a large pizza, is that correct? (entity confirmation)

$\mathrm{U}$ : actually make it small (user correction)

A: You wanted a small pizza, is that correct?

$\mathrm{U}$ : yes

A: What would you like on your pizza?

$\mathrm{U}$ : olives tomatoes and green peppers with thin crust and extra cheese (multi-value slots, overcooperative user)

A: So far I have a small olives, tomatoes, green peppers pizza on thin crust with extra cheese. Is that correct? (context carryover)

$\mathrm{U}$ : yes

A: Great! I placed your order for a total of $\$ 5.99$.

Table 3: A seed conversation with Pizzabot

\section{Case Study 2: Ticketbot}

To showcase the end-customer impact of Alexa Conversations, we built a movie ticket-booking experience which we call Ticketbot. Ticketbot allows a user to browse and book movie tickets. Users can browse currently playing movies by various search 
criteria like date, time, location, theater and movie title. They can specify one or more search criteria either within a single turn or across multiple turns. After finding their choice of movie and theater, users can select a particular showtime, provide booking details like number of tickets and finally confirm booking. The experience was built based on the information provided by the developer. This is a complex experience with 10 APIs, 28 entity types, 10 NLG responses and 35 seed dialogues all provided as an input to the system. This experience was implemented using live APIs that were provided by the developers and thus the users were able to actually achieve their goals and complete ticket-booking transactions.

\subsection{Evaluation}

To evaluate our models, we collected data using a Wizard-of-Oz (WOZ) framework (Asri et al., 2017). These collected dialogues were then annotated by a team of professional annotators using the Dialogue Markup Language. Annotators tagged entities, API and NLG calls and unsupported requests. This is a challenging task and we adopted various methods like inter-annotator agreement and random vetting to ensure high data annotation quality. The test set contained 50 dialogues with an average length of 5.74 turns.

We measure the F1 scores for spans of entities to evaluate NER performance. We also measure the accuracy for action prediction (AP) and full action signature prediction (ASP). The latter metric reflects the performance of both the $\mathrm{AP}$ and $\mathrm{AF}$ models combined: an action signature is counted as correct when both the action and all the corresponding arguments are predicted correctly. We compute these metrics per turn given fixed dialogue context from previous turns, where a turn can contain one user action and multiple agent actions (multiple api calls, nlg call, wait for user action). Turn-level ASP accuracy most closely reflects the user experience when interacting with the skill. Overall, the system has reasonably high turn-level action signature prediction accuracy, with relatively few failures. We discuss some common failure patterns in 6.2.

We evaluate the proposed dialogue simulation method to establish the impact of this novel component. To do so, we train models with data generated using different simulation approaches and compare their performance on the test set. The baseline approach, Base sampler from (Lin et al., 2020),

\begin{tabular}{ccc}
\hline $\begin{array}{c}\text { NER Span } \\
\text { Relative F1 }\end{array}$ & $\begin{array}{c}\text { AP Relative } \\
\text { Accuracy }\end{array}$ & $\begin{array}{c}\text { ASP Relative } \\
\text { Accuracy }\end{array}$ \\
\hline$+18.50 \%$ & $+20.92 \%$ & $+52.80 \%$ \\
\hline
\end{tabular}

Table 4: Relative NER span F1-score, AP accuracy and ASP accuracy on Ticket Booking (TB) test set, averaged over 5 runs.

simply resamples dialogues that are identical in logical structure to the seed dialogues. It generates no new dialogue flows but does add language variations via sampling from developer-provided catalogs and user utterance templates. We observe that models trained on data generated with Sec. 3 significantly outperform the models trained on data generated with baseline as shown in Table 4 .

\subsection{Error Analysis}

We conduct an error analysis of our models on the TicketBot test set to investigate performance across different tasks. We showcase a few common error patterns in this section.

\subsubsection{NER}

We notice that NER model struggles to make correct predictions when the slot value is out of the catalogue vocabulary. As we use fixed slot catalogues during dialogue simulation, it is a difficult task for NER to generalize when real API calls return unseen values. We see that using dynamic catalogue feature significantly improves NER performance, particularly for Movie slot. Dynamic catalogues store entities mentioned in system's responses and thus dynamic catalogue feature provides a strong signal to NER when the user later mentions one of those entities. In addition to exact match, the feature also fires for fuzzy matches leading to higher recall without significant drop in precision. Note that, NER model is not run on system utterances; the entities are tagged by the developer in the response NLG templates. Table 5 shows one example in which "A Star Is Born" is not recognized as Movie without this feature. Overall, by adding dynamic catalogue feature, NER improves by $+11.26 \%$ on the Movie slot.

\subsubsection{ASP}

We find that Argument Filling Model makes mistakes when deciding whether or not to carryover a particular argument. In most such failures, either behavior (carryover or not carryover) is logically correct. However, the user might have a specific be- 
U: give me [sunday $\mid$ Date] showtimes

A: I found [A Star Is Born $\mid$ Movie] playing at

... (mentioned movie)

$\mathrm{U}$ : is [captain marvel $\mid$ Movie] playing [sunday $\mid$ Date]

A: I found showtimes for [Captain Marvel Movie] at ...

$\mathrm{U}$ : is it playing anywhere else

A: Sorry, I could not find any matching showtimes

U: huh ok what are the showtimes for a star is born again

...

Table 5: A partial dialogue from Ticketbot test set showing NER mistake

havior in mind, while the model chooses the other path. For example, in Table 6, AF model carryovers theater and date information, while the particular user wanted to know showtimes at all nearby theaters. As evident, this case is ambiguous as both carrying over and not carrying over the theater and date arguments is reasonable. To define the correct carryover behavior, we advise application developers to provide a few examples demonstrating the carryover behavior for each of their use cases. These examples then bias the dialogue simulator to generate data with the desired carryover behavior.

$\mathrm{U}$ : What is playing at Century Cinemas tomorrow?

A: call: FindMovies(theater $="$ Century Cinemas", date ="tomorrow") -> show0

A: Cold Pursuit is playing at Century Cinemas 16 at 8 PM tomorrow

$\mathrm{U}$ : What are the showtimes for Joker?

A: call: FindMovies(movie="Joker", theater $=$ "Century Cinemas", date $=$ "tomorrow") $->$ showl

...

Table 6: A partial dialogue from Ticketbot test set showing argument carryover mistake

\section{Conclusions}

We presented Alexa Conversations, a novel datadriven and data-efficient approach for building goal-oriented conversational experiences. Our proposed system significantly reduces developer bur- den while still allowing them to build robust experiences. We envision that this system will be used by a wide variety of developers who only need to provide seed dialogues and action schema to build conversational experiences ${ }^{1}$.

We expect our system to mature in the following directions in future. We aim to reduce developer requirements for providing NLG responses by introducing a statistical NLG system. We will also develop robust mechanisms for incorporating developer feedback through supervised and semisupervised methods to improve the performance of our simulator and modeling components.

\section{References}

Layla El Asri, Hannes Schulz, Shikhar Sharma, Jeremie Zumer, Justin Harris, Emery Fine, Rahul Mehrotra, and Kaheer Suleman. 2017. Frames: A corpus for adding memory to goal-oriented dialogue systems. arXiv preprint arXiv:1704.00057.

Danqi Chen. 2018. Neural Reading Comprehension and Beyond. Ph.D. thesis, Stanford University.

Heriberto Cuayáhuitl, Steve Renals, Oliver Lemon, and Hiroshi Shimodaira. 2005. Human-computer dialogue simulation using hidden markov models. In IEEE Workshop on Automatic Speech Recognition and Understanding, 2005., pages 290-295. IEEE.

Jacob Devlin, Ming-Wei Chang, Kenton Lee, and Kristina Toutanova. 2018. Bert: Pre-training of deep bidirectional transformers for language understanding. arXiv preprint arXiv:1810.04805.

Maryam Fazel-Zarandi, Shang-Wen Li, Jin Cao, Jared Casale, Peter Henderson, David Whitney, and Alborz Geramifard. 2017. Learning robust dialog policies in noisy environments. 1st Workshop on Conversational AI at NIPS.

Shuyang Gao, Abhishek Sethi, Sanchit Aggarwal, Tagyoung Chung, and Dilek Hakkani-Tur. 2019. Dialog state tracking: A neural reading comprehension approach. Proceedings of the 20th Annual SIGdial Meeting on Discourse and Dialogue (SIGDIAL).

Izzeddin Gur, Dilek Zeynep Hakkani, Gökhan Tür, and Pararth Shah. 2018. User modeling for task oriented dialogues. 2018 IEEE Spoken Language Technology Workshop (SLT), pages 900-906.

Chien-Wei Lin, Vincent Auvray, Daniel Elkind, Arijit Biswas, Maryam Fazel-Zarandi, Nehal Belgamwar, Shubhra Chandra, Matt Zhao, Angeliki Metallinou,

\footnotetext{
${ }^{1}$ iRobot (https://tinyurl.com/y5pjp3xn), BigSky (https://tinyurl.com/y2ejvd3z) and Art Museum (https://tinyurl.com/y3umpqo2) are some of the external skills that have already been built using Alexa Conversations
} 
Tagyoung Chung, et al. 2020. Dialog simulation with realistic variations for training goal-oriented conversational systems. 1st Workshop on Human in the Loop Dialogue Systems at Neurips.

Bing Liu, Gokhan Tur, Dilek Hakkani-Tur, Pararth Shah, and Larry Heck. 2017. End-to-end optimization of task-oriented dialogue model with deep reinforcement learning. arXiv:1711.10712.

X. Ma and E. Hovy. 2016. End-to-end sequence labeling via bi-directional lstm-cnns-crf. In Proc. of the 54th Annual Meeting of the ACL (ACL) 2016.

Matthew E Peters, Mark Neumann, Mohit Iyyer, Matt Gardner, Christopher Clark, Kenton Lee, and Luke Zettlemoyer. 2018. Deep contextualized word representations. arXiv preprint arXiv:1802.05365.

Olivier Pietquin. 2005. A framework for unsupervised learning of dialogue strategies. Presses univ. de Louvain.

Olivier Pietquin and Thierry Dutoit. 2006. A probabilistic framework for dialog simulation and optimal strategy learning. IEEE Transactions on Audio, Speech, and Language Processing, 14(2):589-599.

Jost Schatzmann, Blaise Thomson, Karl Weilhammer, Hui Ye, and Steve Young. 2007. Agenda-based user simulation for bootstrapping a pomdp dialogue system. In Human Language Technologies 2007: The Conference of the North American Chapter of the Association for Computational Linguistics; Companion Volume, Short Papers, pages 149-152. Association for Computational Linguistics.

Pararth Shah, Dilek Hakkani-Tür, Gokhan Tür, Abhinav Rastogi, Ankur Bapna, Neha Nayak, and Larry Heck. 2018. Building a Conversational Agent Overnight with Dialogue Self-Play. (i).

Tsung Hsien Wen, David Vandyke, Nikola Mrkšíc, Milica Gašíc, Lina M. Rojas-Barahona, Pei Hao Su, Stefan Ultes, and Steve Young. 2017. A network-based end-to-end trainable task-oriented dialogue system. 15th Conference of the European Chapter of the Association for Computational Linguistics, EACL 2017 - Proceedings of Conference, 1:438-449.

Jason D Williams, Kavosh Asadi, and Geoffrey Zweig. 2017. Hybrid code networks: practical and efficient end-to-end dialog control with supervised and reinforcement learning. arXiv preprint arXiv:1702.03274.

Kyle Williams. 2019. Neural lexicons for slot tagging in spoken language understanding. In 2019 Annual Conference of the North American Chapter of the Association for Computational Linguistics (NAACL '19).

Steve J Young. 2000. Probabilistic methods in spoken-dialogue systems. Philosophical Transactions of the Royal Society of London. Series A. Mathematical, Physical and Engineering Sciences, 358(1769):1389-1402. 